Sharif University of Technology
Scientia Iranica
SCIENTIA $\quad \begin{gathered}\text { Transactions D: Computer Science \& Engineering and Electrical Engineering } \\ \text { whw.scientiairanica.com }\end{gathered}$

\title{
A multi-objective heuristic method for optimal design of HTS fault current limiters
}

\author{
R. Sharifi ${ }^{\mathrm{a}, \mathrm{b}}$ and H. Heydari $\mathrm{a}^{\mathrm{a}, *}$ \\ a. Center of Excellence for Power System Automation and Operation, Electrical Engineering Department, Iran University of \\ Science and Technology (IUST), Tehran, Iran. \\ b. Department of Electrical Engineering, West Tehran Branch, Islamic Azad University, Tehran, Iran.
}

Received 27 July 2014; accepted 20 February 2016

\section{KEYWORDS}

Multi-objective decision making; Multi-objective simulated annealing; High temperature superconductors; Superconducting fault current limiter.

\begin{abstract}
The optimization algorithm for High-Temperature Superconducting (HTS) Fault Current Limiters (FCL) normally includes some non-commensurable criteria or objectives such as cost, limiting factor, thermal stresses, and mechanical stresses that should be minimized or maximized concurrently in a protracted optimization process. It can be performed only by tools which firstly describe the HTSFCL precisely and predict its limitation behavior and then select the optimum design using optimization algorithm. Multi-Objective Decision Making (MODM) is one of the most widely used decision techniques in the business and engineering worlds. In the MODM problems, there are several objectives of the system concurrently optimized, and a solution set, i.e. the Pareto front, is usually obtained instead of a real optimal solution. This paper explains in details the combination models of HTSFCL as a component in PSCAD/EMTDC and presents the optimization algorithm based on a new approach of normalized multi-objective simulated annealing.
\end{abstract}

(C) 2016 Sharif University of Technology. All rights reserved.

\section{Introduction}

The High-Temperature Superconducting (HTS) Fault Current Limiter (FCL) is one of the main solutions proposed to reduce high fault currents in rapidly growing electrical systems. The advantages of HTSFCL, such as automatic fault current sensing, automatic recovering, and faster limiting operations, are expected to be the unique countermeasure to solve the drawbacks of the other limiting devices.

The superconducting state is defined by three important factors: critical temperature $\left(T_{c}\right)$, critical magnetic field $\left(H_{c}\right)$, and critical current density $\left(J_{c}\right)$. Each of these parameters is very dependent on the other two properties present. The limiting operation

\footnotetext{
*. Corresponding author. Fax: +98 21 r7208123 E-mail address: Heydari@iust.ac.ir (H. Heydari)
}

is a multilateral interaction between the fault current, temperature, magnetic field, and current dependent impedance in addition to other specifications in the external power system.

Hence, for an optimal design of parameters and dimensions, a comprehensive model describing HTSFCL behavior for different statuses of power system is needed [1]. The optimization algorithm for HTSFCL generally includes some non-commensurable criteria or objectives such as cost, limiting factor, and thermal and mechanical stresses being minimized or maximized simultaneously in a protracted process. This can be accomplished only by tools being capable of describing the HTSFCL and precisely predicting its limitation behavior followed by selecting an optimum design via optimization algorithm.

Hence comes the need for an advanced technique for optimization concepts based on physical and elec- 
trical properties of the HTSFCL. However, as far as the authors are aware, such software does not exist among commercial tools.

Various types of HTSFCL devices exist, including normal resistive, flux flow resistive, magnetic shield inductive, transformer inductive, DC reactor, fluxlock, and saturated core [2-4]. Among these FCLs, inductive-type superconducting fault current limiters (LSFCL) have made themselves prominent because of large design flexibility due to turn ratio and multi benefits of not needing current lead causing isolation between FCL and power transmission line, heat loss reduction (lower resistance), and smaller impedance due to inductive impedance of power system $[5,6]$.

However, the optimization process can readily be used it in any types other than that described above, without departing from the spirit of the concept.

The LSFCL generally consists of a primary copper coil and a secondary superconductor cylinder/coil wound around a closed or open magnetic iron core. In the shielded core LSFCL, superconducting cylinder is fixed between copper coil and magnetic core; therefore, screen currents cause no flux penetration into iron core in normal condition. In the transformer type, secondary winding is a cooper coil shortened via a superconducting component, resulting in nearly zero impedance from primary side. In the event of a fault, a superconducting to normal $(S / N)$ transition occurred in both LSFCLs and reflected limiting impedance appeared in the primary side [5].

For non-consistent objectives, multi-objective optimization algorithms attempt to find proper solutions in Multi-Objective Decision Making (MODM) problems. In general, such problems have multiple solutions that constitute the Pareto optimal set instead of a real optimal solution. Considering all objectives, these solutions are optimal in a sense that no other solutions in the search space are superior to one another. One of the most frequent methods in obtaining Pareto solutions has been realized by the application of heuristic methodologies, such as Genetic Algorithms (GA), Evolutionary Algorithms (EA), and Simulated Annealing (SA). These methods are based on the phenomena of their principles being observed in nature, from which SA is a robust algorithm for solving single-objective optimization problems [7]. The major advantage of SA over the other methods is the uniqueness of its non-global trapped local minima. The algorithm employs a random search which accepts not only the changes that decrease objective function, but also some changes that increase it. D. Geman and S. Geman [8] proposed that the designed algorithm converges to a global optimum when the annealing process is accomplished sufficiently slowly. A number of comparisons of the multi-objective SA algorithms have already been reported [9]. SA optimization algorithms in single- or multiple-objective forms have also been used for designing some superconducting devices such as microwave filter [10], magnet [11], energy storage device [12], generator [13], and resistive fault current limiter $[14,15]$ based on their capability to find the global optimum.

This paper attempts to supplement our previous report on the normalized prioritized multi-objective simulated annealing [14] by presenting a new optimization algorithm being improved by a new normalization method. This was accomplished by performing numerical analysis of electrical and thermal behavior of the LSFCL in a feasible PSCAD/EMTDC model so as to select the optimized design via the proposed algorithm.

\section{Inductive-type superconducting fault current limiter}

As far as limiting impedance is concerned, HTSFCLs have been classified into resistive type (RSFCL), inductive type (LSFCL), and hybrid type (HSFCL) [16]. However, some of the previous reports have divided the LSFCLs into quench and non-quench types. The former, in which $S / N$ transition occurs, includes the magnetic shielding type, the transformer type, and the ring type; while the latter includes the saturation reactor and dc reactor types. Except negligible difference during normal system operation, LSFCLs all act similarly in fault condition. Therefore, a common model can be considered for the current limiting regime.

In the quench-type LSFCL, generally, the primary copper coil and a secondary superconductor tube/coil were wound around a closed/open magnetic iron core. In the shielded core LSFCL, superconducting tube (shown in Figure 1) is fixed between copper coil and magnetic core; thereby, the screen currents would prevent flux penetration into iron core during normal operation. Another version of LSFCL is the transformer type with copper secondary winding shortened by an

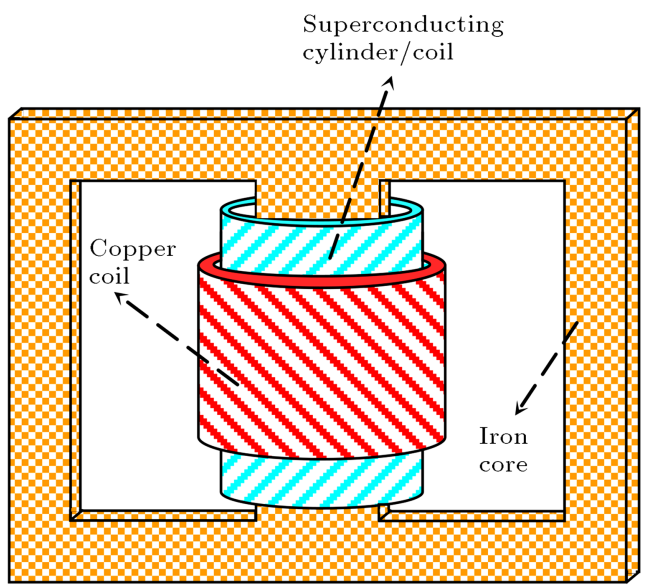

Figure 1. Magnetic shield type superconductive fault current limiter. 


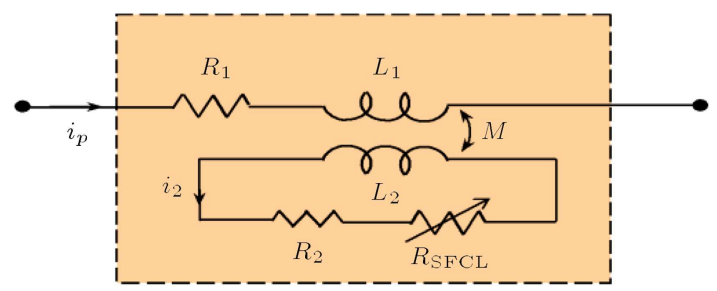

(a)

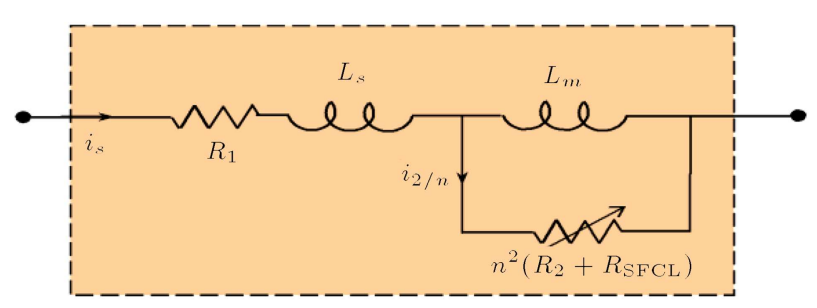

(b)

Figure 2. (a) Common model of LSFCL. (b)

Corresponded equivalent circuit.

HTS element "resistor $\left(R_{\mathrm{SFCL}}\right)$ " placed in cryostat and cooled down by liquid nitrogen via a current lead, giving almost zero impedance on the primary side. Figure 2 shows the corresponding common equivalent circuit for the aforementioned LSFCLs in which $R_{2}$ does only exist for considering transformer (secondary copper winding). In this figure, $i_{p}$ and $i_{s}$ are the primary and corresponding secondary currents, respectively. Although the superconductor materials used in the transformer type are fewer, there is an effective magnetic coupling that causes more core loss and secondary copper loss than magnetic shielded type [2].

In the event of a fault occurring, the increasing current exceeds the critical value of the HTS component; then, the resistance of the secondary winding is reflected into the primary circuit; and the magnetic flux penetrates the iron core, increasing the impedance of limiter $[5,17]$.

Considering Figures 1 and 2, under fault conditions, the high current in the copper coil exceeds the shielding capability of the superconductor tube, leading to a jump in impedance that is flux penetration into the iron core. As a result, the limiting impedance is formed by the magnetizing inductance of the transformer in parallel to the resistance of the superconducting tube, reflected on the primary side by the factor of $\left(N_{1} / N_{2}\right)^{2}$. The design parameters mainly determine the resistive or inductive characteristic of the limiting impedance.

The advantage of LSFCLs is that the secondary winding of the magnetic shield and transformer types can be made of either a single or few HTS rings. The working principle of this type of limiter is based on the field screening effect of the HTS, which drives the magnetic core to a zero flux condition in superconducting state [18]. This type of LSFCL is similar to the transformer type with no secondary copper winding. Therefore, a common model (Figure 2) can be considered based on the aforementioned behavior in which electrical equivalent circuit of transformer is accompanied with a variable load [19].

Different types of LSFCLs have some advantages including physical separation of electric circuit and superconducting material, lower voltage drop across the superconducting element during fault conditions, and possibility of making the superconducting coil in simple BSCCO rings or cylinders [20]. Likewise, the secondary coil can be made from the new generation of economical superconducting wires named YBCO coated conductors [21].

In these figures, $R_{1}, R_{2}, L_{1}, L_{2}, M, L_{s}$, and $L_{m}$ are the resistance of primary and secondary windings (for magnetic shield type $R_{2}=0$ ), the self-inductance of primary and secondary windings, the mutual inductance between primary and secondary windings, the stray inductance of primary winding, and the magnetizing inductance of transformer, respectively. The turn ratio of transformer is denoted by $n=\left(N_{1}\right)$ $N_{2}$ ).

By considering $h, r_{c}, r_{p}$, and $r_{s}$ as the height of the windings, core radius, primary radius, and radius of secondary coil/tube, respectively, the inductances can be calculated as [19]:

$$
\begin{aligned}
& L_{1}=\frac{\pi \mu_{0} n^{2}}{h}\left\{r_{p}^{2}+\left(\mu_{r}-1\right) r_{c}^{2}\right\}, \\
& L_{2}=\frac{\pi \mu_{0}}{h}\left\{r_{s}^{2}+\left(\mu_{r}-1\right) r_{c}^{2}\right\}=\frac{M}{n}, \\
& M=\frac{\pi \mu_{0} n}{h}\left\{r_{s}^{2}+\left(\mu_{r}-1\right) r_{c}^{2}\right\},
\end{aligned}
$$

where, $\mu_{r}$ is the relative permeability of the core. If the transformer model of LSFCL is simplified by Figure 2(b), the stray and magnetizing inductances can be written as:

$$
\begin{aligned}
& L_{s}=(1-k) L_{1}=L_{1}-n^{2} L_{2}=\frac{\pi \mu_{0} n^{2}}{h}\left(r_{p}^{2}-r_{s}^{2}\right), \\
& L_{m}=k L_{1}=n M=\frac{\pi \mu_{0} n^{2}}{h}\left\{r_{s}^{2}+\left(\mu_{r}-1\right) r_{c}^{2}\right\} .
\end{aligned}
$$

The change of the resistance of HTS can be estimated by a non-linear voltage-current characteristic, including three portions corresponding to the flux creep, flux flow, and normal state described in [22]. Moreover, the total power loss of HTS is the sum of Joule loss and the ac loss:

$$
P_{s c}=P_{j}+P_{a c} \text {. }
$$

Thus, generating heat and HTS temperature can be calculated by integration of power loss [14]:

$$
T=\frac{1}{C_{p}}\left(\int P_{s c} d t-\frac{P_{c s} \Delta t}{V_{s c}}\right)+T_{0},
$$


where, $P_{c s}$ is the cooling system power, and $V_{s c}$, $\Delta_{t}$, and $C_{p}$ are the volume of the HTS coil, fault duration time, and specific heat of HTS material and stabilizer. In general, stabilizer of HTS, which is made of metals (i.e., silver, steel, copper, etc.), acts as a shunt resistance bypassing the current and heat during quench time. The stabilizer helps to avoid destructive hot spots caused by local quench [23].

To calculate the ac loss, Bean model [24] (used by [19]) or Norris model [25] can be used as:

$$
\begin{aligned}
& P_{a c}=\frac{8 \sqrt{2}}{3} f \mu_{0} \frac{r_{s}}{h^{2}} \frac{\left(n i_{p}\right)^{3}}{j_{c}} \\
& P_{a c}=\frac{f \mu_{0}}{\pi} \frac{i_{s}}{j_{c}}\left[\left(1-\frac{i_{s}}{i_{c}}\right) \ln \left(1-\frac{i_{s}}{i_{c}}\right)+\left(2-\frac{i_{s}}{i_{c}}\right) \frac{i_{s}}{2 i_{c}}\right],
\end{aligned}
$$

where, $f, i_{p}, i_{s}, i_{c}$, and $j_{c}$ are frequency, primary current, secondary current, critical current, and critical current density of HTS, respectively. In addition to ac loss, the core losses and current lead loss must also be taken into account for transformer type. Since the secondary side is short-circuited by a superconducting element, the core loss is very low, that is $0.01 \%-0.1 \%$ of transformer rating [20]. Furthermore, the minimum loss due to current lead (if exists) is estimated by Eq. (10) assuming temperature-dependent coefficients $\kappa$ and $\rho$ as average values of thermal conductivity and resistivity, respectively [26]:

$$
P_{c l}=2 n i_{p} \sqrt{\rho \kappa \Delta T} \text {. }
$$

\section{Multi-objective optimization method}

Generally, engineering design problems require a simultaneous optimization for several incompatible objectives. To study the trade-offs that exist between these conflicting design objectives/goals and to explore design options, one needs to formulate the optimization problem with multiple-objective decision making techniques. These optimization methods seek some optimum designs that attain the multiple objectives as closely as possible while strictly satisfying constraints [27].

In a multi-objective optimization problem consisting of $N_{v}$ variables, $N_{f} \geq 2$ objective functions are optimized subject to $N_{c}$ constraints:

$$
\operatorname{Min}\left\{f_{i}\left(x_{j}\right)\right\} \forall i=1,2, \ldots, N_{f} \text { and } j=1,2, \ldots, N_{v}
$$

Subject to:

$$
g_{k}\left(x_{j}\right) \leq 0 \quad k=1,2, \ldots, N_{c}
$$

A point $X^{\prime}=\left\{x_{1}^{\prime}, x_{2}^{\prime}, \ldots, x_{N_{v}}^{\prime}\right\}$ is defined as being
Pareto-optimal if and only if there is no other point, $X=\left\{x_{1}, x_{2}, \ldots, x_{N_{v}}\right\}$, which can dominate it:

$$
\begin{cases}\forall i=1,2, \ldots, N_{f} & f_{i}(X) \leq f_{i}\left(X^{\prime}\right) \\ \forall i & f_{i}(X)<f_{i}\left(X^{\prime}\right)\end{cases}
$$

One of the most useful techniques for solving complicated optimization problems, the simulated annealing, was introduced by Kirkpartick et al. [7]. This technique was originally inspired by the formation of crystals in solids during cooling. The method itself has a direct similarity with thermodynamics, specifically with the way that liquids freeze and crystallize, or metals cool and anneal. In order to simulate the annealing process of metals, the material is considered as a system of particles. The probability of a particle being in a specific energy level $(\Delta E)$ is expressed by the Boltzmann probability distribution:

$$
P(\Delta E)=\exp \left(-\frac{\Delta E}{K T}\right),
$$

where, $K$ and $T$ are the Boltzmann constant and temperature, respectively. Kirkpatrick created a variation of the classical local search method with an important difference. According to some probability function based on Boltzmann probability distribution, the inferior moves are accepted. Starting the algorithm, almost all the inferior moves are accepted so that the procedure operates as a random search. However, as the temperature decreases, fewer inferior moves are accepted; but the algorithm still has the ability to escape from local optima [28].

In the recent years, several SA-based algorithms, e.g. Weight-Based Multi-Objective Simulated Annealing (WMOSA) [29], Pareto dominant-based multi-objective simulated annealing (PDMOSA) [30], Archived Multi-Objective Simulated Annealing (AMOSA) [31], Prioritized Multi-Objective Simulated Annealing (PMOSA) [28], and Dominance Cost variant Multi-Objective Simulated Annealing (DCMOSA) [32], have been presented for optimization of engineering problems.

In PMOSA, the algorithm satisfies the given priority of objectives by assigning a different initial temperature for each objective. Furthermore, contentment of the constraints is considered as a top priority being incorporated as additional terms in the first objective function:

$$
F_{1}(X)=f_{1}(X)+b \sum_{k=1}^{N_{c}} \operatorname{Max}\left\{0, g_{k}(X)\right\},
$$

where $b$ is used to change the relative importance of additional terms and $N_{c}$ is number of constraints. Regarding the importance priority of objective functions, 


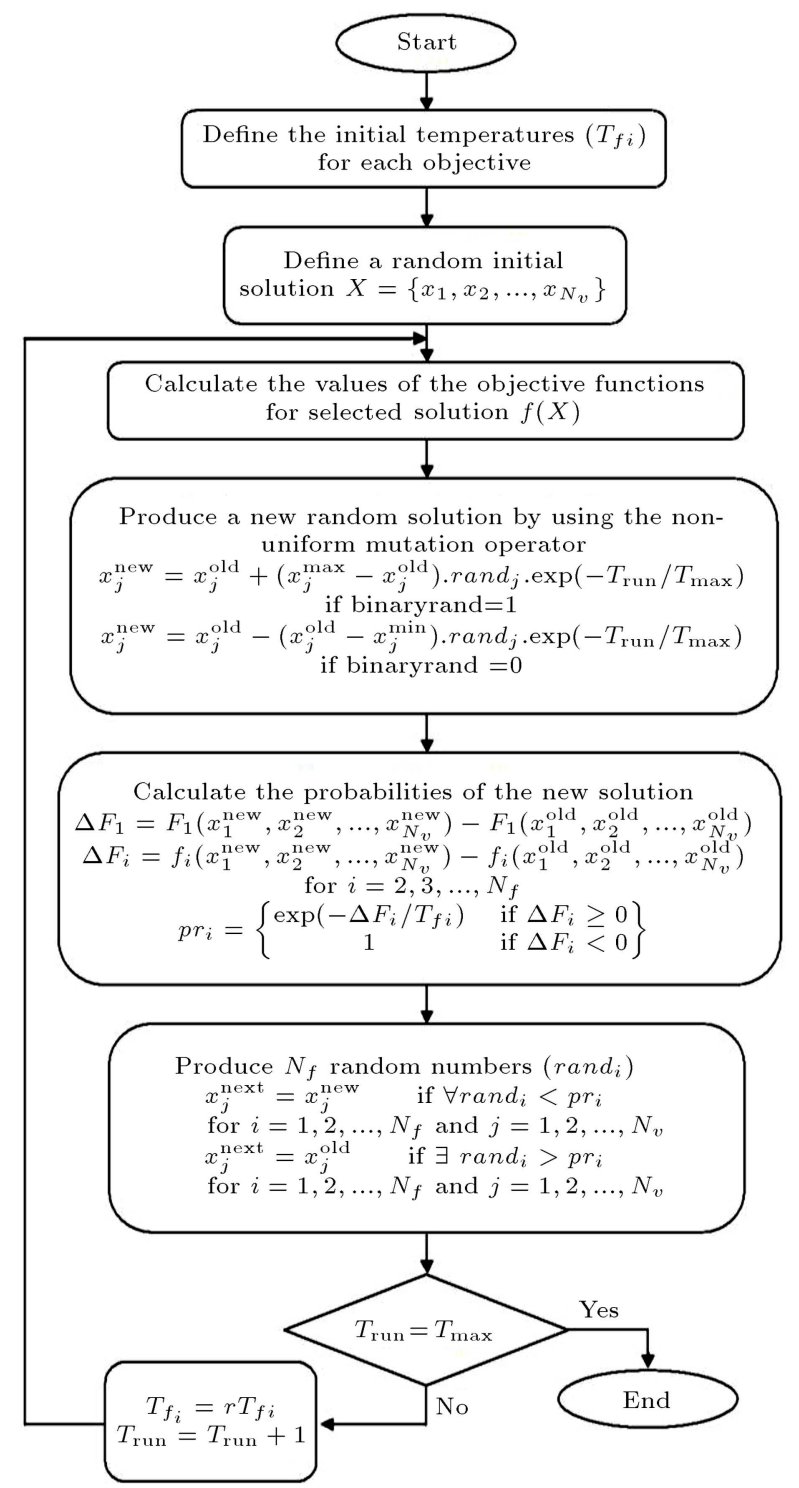

Figure 3. PMOSA optimization algorithm.

$N_{f}$ temperatures are used to obtain Pareto front. The steps of the PMOSA algorithm can be summarized as shown in Figure 3.

In this algorithm, if the variables and objective functions are normalized to compare and combine different types of parameters, the normalized PMOSA (henceforth, NPMOSA) is obtained. This improvement is very important; because in optimizing design of an HTSFCL, many parameters and functions have not the same dimensions and magnitude, since the probabilities of acceptance are influenced by the values of the objective functions. Thus, using only various initial temperatures for objective functions cannot be feasible.

In NPMOSA algorithm, differential of probabilities $\left(\Delta F_{i}\right)$ for any function is normalized by being divided to the present value. Consequently, they are comparable to each other:

$$
\begin{aligned}
& \Delta F_{i}^{\text {norm }}=\left(f_{i}\left(x_{j}^{\text {new }}\right)-f_{i}\left(x_{j}^{\text {old }}\right)\right) / f_{i}\left(x_{j}^{\text {new }}\right) \\
& \quad \text { for } i=2,3, \ldots, N_{f} .
\end{aligned}
$$

This relation can be summarized as:

$$
\Delta F_{i}^{\text {norm }}=1-\frac{f_{i}\left(x_{j}^{\text {old }}\right)}{f_{i}\left(x_{j}^{\text {new }}\right)} \quad \text { for } i=2,3, \ldots, N_{f} .
$$

However, this normalization technique is not adequately straightforward for the first objective function $\left(\Delta F_{1}\right)$. Initially, the constraints in Eq. (14) must be normalized, which are divided by a constant, e.g. their initial value. After that, they are necessary to be similar with the objective function multiplied by its initial value:

$$
\begin{aligned}
F_{1}\left(x_{j}\right)= & f_{1}\left(x_{j}\right)+b \sum_{k=1}^{N_{c}} \operatorname{Max} \\
& \left\{0,\left|\frac{f_{1}\left(x_{j}^{\text {initial }}\right)}{g_{k}\left(x_{j}^{\text {initial }}\right)}\right| g_{k}\left(x_{j}\right)\right\} .
\end{aligned}
$$

In the next step, the differential of probability, assuming $N_{c}$ constraints, can be written as:

$$
\Delta F_{1}=\Delta f_{1}\left(x_{j}\right)+b \Delta G\left(x_{j}\right)
$$

where:

$$
\begin{aligned}
\Delta f_{1}\left(x_{j}\right)= & f_{1}\left(x_{j}^{\text {new }}\right)-f_{1}\left(x_{j}^{\text {old }}\right) \\
\Delta G\left(x_{j}\right)= & \sum_{k=1}^{N_{c}} \operatorname{Max}\left\{0,\left|\frac{f_{1}\left(x_{j}^{\text {initial }}\right)}{g_{k}\left(x_{j}^{\text {initial }}\right)}\right| g_{k}\left(x_{j}^{\text {new }}\right)\right\} \\
& -\sum_{k=1}^{N_{c}} \operatorname{Max}\left\{0,\left|\frac{f_{1}\left(x_{j}^{\text {initial }}\right)}{g_{k}\left(x_{j}^{\text {initial }}\right)}\right| g_{k}\left(x_{j}^{\text {old }}\right)\right\}
\end{aligned}
$$

Eq. (21) can be normalized if divided by $f_{1}\left(x_{j}^{\text {new }}\right)$ :

$$
\Delta F_{1}^{\text {norm }}=1-\frac{f_{1}\left(x_{j}^{\text {old }}\right)}{f_{1}\left(x_{j}^{\text {new }}\right)}+\frac{b}{f_{1}\left(x_{j}^{\text {new }}\right)} \Delta G\left(x_{j}\right) .
$$

Considering the proposed phases, this method can be named First Difference Last Normalizing (FNLD). In the contrary method, another way to achieve normalized function is to calculate the differential of probability after normalizing the objective function and constraints divided by their related value:

$$
\begin{aligned}
F_{1}^{\text {norm }}\left(x_{j}^{\text {old }}\right)= & \frac{f_{1}\left(x_{j}^{\text {old }}\right)}{f_{j}\left(x_{j}^{\text {new }}\right)} \\
& +b \sum_{k=1}^{N_{c}} \operatorname{Max}\left\{0, \frac{g_{k}\left(x_{j}^{\text {old }}\right)}{\left|g_{k}\left(x_{j}^{\text {new }}\right)\right|}\right\},
\end{aligned}
$$




$$
\begin{aligned}
F_{1}^{\text {norm }}\left(x_{j}^{\text {new }}\right)= & 1+b \sum_{k=1}^{N_{c}} \operatorname{Max}\left\{0, \frac{g_{k}\left(x_{j}^{\text {new }}\right)}{\left|g_{k}\left(x_{j}^{\text {new }}\right)\right|}\right\}, \\
\Delta F_{1}^{\text {norm }}=1- & \frac{f_{1}\left(x_{j}^{\text {old }}\right)}{f_{1}\left(x_{j}^{\text {new }}\right)}+b \Delta G^{\text {norm }}\left(x_{j}\right), \\
\Delta G^{\text {norm }}\left(x_{j}\right)= & \sum_{k=1}^{N_{c}} \operatorname{Max}\left\{0, \frac{g_{k}\left(x_{j}^{\text {new }}\right)}{\left|g_{k}\left(x_{j}^{\text {new }}\right)\right|}\right\} \\
& -\sum_{k=1}^{N_{c}} \operatorname{Max}\left\{0, \frac{g_{k}\left(x_{j}^{\text {old }}\right)}{\left|g_{k}\left(x_{j}^{\text {old }}\right)\right|}\right\} .
\end{aligned}
$$

In a similar way, the recent method can be named First Difference Last Normalizing (FNLD). In a case study, these methods were found to have higher performance in an HTSFCL design.

\section{Performance of the proposed NPMOSA methods}

To evaluate the proposed NPMOSA approaches in design of superconducting fault current limiter, a model in which an LSFCL is located at an outgoing feeder in a $20 \mathrm{kV}$ distribution substation (single phase $11.5 \mathrm{kV}$ ) is considered. A simplified model is shown in Figure 4 as a single-phase equivalent circuit supposing a three-phase short-circuit fault occurring close to the substation.

A component in PSCAD/EMTDC environment was defined to achieve the combined electrical and thermal model of LSFCL. Simulations were carried out with a fault occurring at $t=100 \mathrm{~ms}$ (phase angle $=0$ ) for maximum overshoot current and cleared after $\Delta t=$ $300 \mathrm{~ms}$ that is a suitable time for operating or re-closing the protection devices. The total simulation time was considered about 4 seconds, which is enough for quenching and restoration of the HTS. The simulation time interval of $10 \mu$ s was sufficient to observe the transient pattern. The characteristics of the system and the selected LSFCL parameters are shown in Table 1.

In order to evaluate the operating characteristic and limiting behaviors of the LSFCL, case simulations based on the sample parameters were carried out with and without the limiter. The feeder peak current in pre-fault state is $390 \mathrm{~A}$ and exceeds $4.5 \mathrm{kA}$ at the

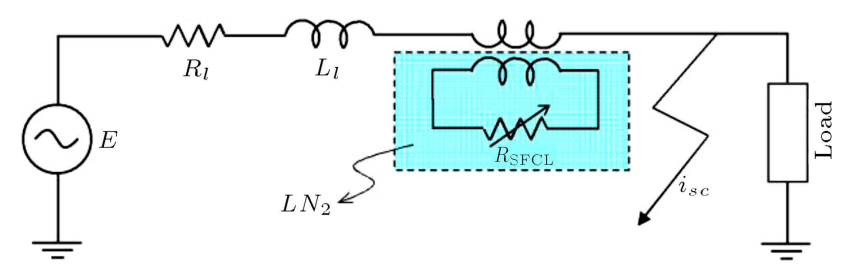

Figure 4. A single-phase equivalent circuit of an electrical system with LSFCL.
Table 1. System parameters in the simulation model.

\begin{tabular}{clc}
\hline Symbol & \multicolumn{1}{c}{ Quantity } & Value \\
\hline$E$ & Phase voltage $(\mathrm{kV})$ & $20 / \sqrt{3}$ \\
$R_{l}$ & Resistance of the line $(\Omega)$ & 1.5 \\
$L_{l}$ & Inductance of the line $(\mathrm{mH})$ & 15 \\
$f$ & System frequency $(\mathrm{Hz})$ & 50 \\
Load & Load power of the system $(\mathrm{MW})$ & 3 \\
$T_{c}$ & Critical temperature for HTS $(\mathrm{K})$ & 90 \\
$T_{0}$ & Temperature of $L N_{2}(\mathrm{~K})$ & 77 \\
$I_{c 0}$ & Critical current in $T=T_{c}(\mathrm{~A})$ & 720 \\
$R_{s h}$ & Shunt resistance to HTS $(\Omega)$ & 0.01 \\
$C_{p}$ & Specific heat of HTS $\left(\mathrm{MJm}{ }^{-3} \mathrm{~K}^{-1}\right)$ & 1 \\
$P$ & Cooling power $(\mathrm{MW})$ & 1.6 \\
$V_{S C}$ & Volume of HTS $\left(\mathrm{m}^{3}\right)$ & $2 \times 10^{-2}$ \\
$A_{S C}$ & Cross section of HTS $\left(\mathrm{m}^{2}\right)$ & $6 \times 10^{-4}$ \\
$h$ & Height of iron core $(\mathrm{m})$ & 1.5 \\
$\mu$ & Relative permeability of core & 65 \\
$r_{c}$ & Radius of iron core $(\mathrm{m})$ & 0.4 \\
$r_{s}$ & Radius of HTS cylinder $(\mathrm{m})$ & 0.52 \\
$r_{p}$ & Radius of copper coil $(\mathrm{m})$ & 0.7 \\
$n$ & Turn ratio of transformer $\left(N_{1} / N_{2}\right)$ & 120 \\
$P_{a c}$ & Maximum AC loss $(\mathrm{W})$ & 22.1 \\
\hline & &
\end{tabular}

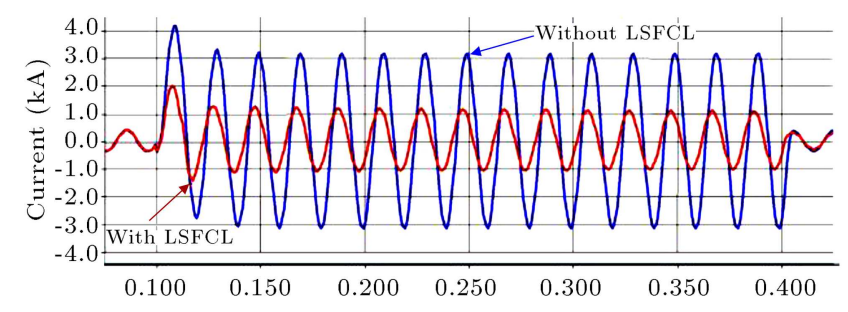

Figure 5. The current limiting performance with LSFCL.

first cycle, and $3.25 \mathrm{kA}$ in steady state without any limiter device. By locating an acceptable (pre-optimum design) LSFCL in the system, the fault current is reduced to $2.1 \mathrm{kA}$ in the flux flow mode and $1.15 \mathrm{kA}$ in normal mode of HTS. The feasibility of the model for limiting the fault current in the study system is shown in Figure 5, while temperature and resistance variations are illustrated in Figure 6.

The current limiting impedance of LSFCL consists of a pure resistance of the HTS and a magnetizing reactance of the magnetic circuit. Based on Eqs. (1) to (5), the estimated stray inductance and calculated magnetizing inductance of the LSFCL are about $8.33 \mathrm{mH}$ and $398 \mathrm{mH}$ with corresponding reactances of $2.616 \Omega$ and $125.3 \Omega$, respectively. The HTS resistance $\left(R_{\text {HTSFCL }}\right)$ exceeds $0.32 \mathrm{~m} \Omega$ and $0.75 \mathrm{~m} \Omega$ in flux flow and normal mode in the fault occasion. These resistances entail corresponding values on the primary side $4.6 \Omega$ and $10.8 \Omega$, respectively. The variations of 


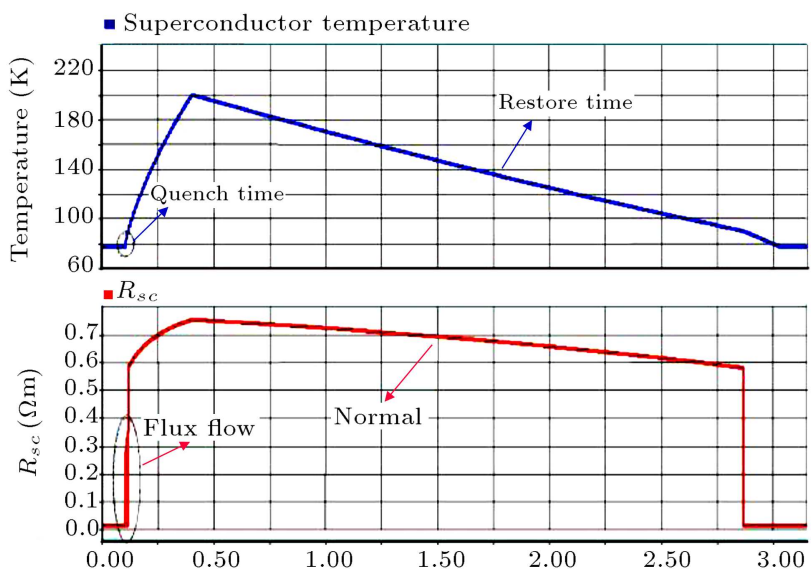

Figure 6. The variations of superconducting temperature and resistance on the fault occasion.

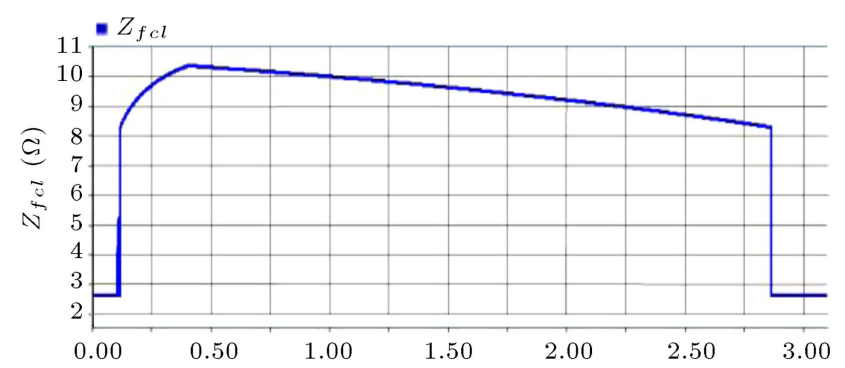

Figure 7. The variation of total limiting impedance.

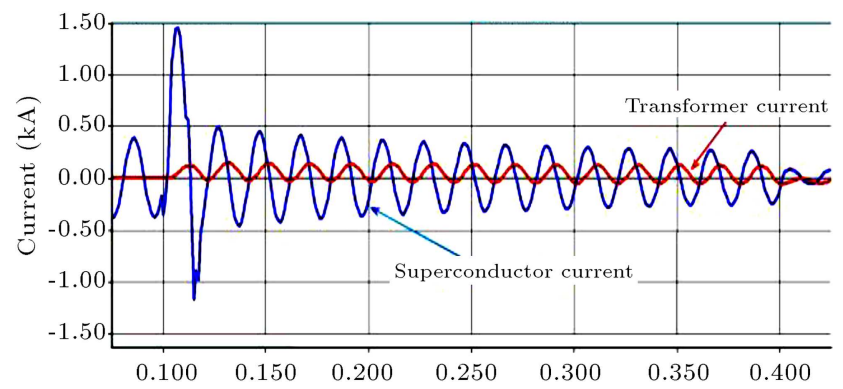

Figure 8. The current of resistive and inductive part.

the total impedance of the LSFCL $\left(X_{m} \| R_{\text {HTSFCL }}\right)$ are shown in Figure 7 . It is shown that the maximum limiting impedance is about $10.43 \Omega$ prior to the fault removal from the feeder.

The variations of the current through the magnetizing reactance of transformer and the HTS during fault state are compared in Figure 8. Considering the two limiting impedances with different natures, an oscillation of $90^{\circ}$ phase difference occurs. It is noticeable that the HTS current, reflected on the primary side, is comparable with reactance current. It can be seen that the HTS element, which lingers in normal mode during restoring time and gives rise to the limiting impedance, remains in the line current path leading to voltage sag on the load side.

As stated before, in order to perform applicable optimization of inductive HTSFCL design, this paper proposes two methods (FNLD and FDLN) for normalizing multi-objective simulating annealing algorithm. The formulation of these methods is described by treating the problem as a multi-objective programming which runs the combined electrical and thermal model several times for different parameters. The problems are concerned with minimizing or maximizing each objective, simultaneously, while accounting for the constraints.

In this study, three objective functions are considered for design of an inductive HTSFCL. The first objective function is maximizing the current limiting factor $(\alpha)$ that is the ratio of the let-through shortcircuit current (i.e., the short circuit current in the absence of a limiting device) to the limited shortcircuit current within the first half cycle [33]. Another one is minimizing the voltage sag $(\Delta E)$ during postfault clearance that is an important index in power quality issue and finally, the last objective function is minimizing the HTSFCL cost $(C)$ proportional to superconductor, copper, and iron volume. These objective functions are varied by electrical, thermal, and dimensional parameters being summarized in Table 1. As the majority of the parameters are usually invariable and dependent on one another or beyond the control of the designer, this study concentrates on superconductor and transformer parameters, i.e. HTS volume $\left(V_{s c}\right)$, transformer turn ratio $(n)$, magnetic permeability $(\mu)$, and core dimensions $\left(r_{c}\right.$ and $\left.h\right)$. Other design parameters have no considerable effect or can be written as selected variables. Hence, the problem can be formulated as:

$$
\begin{aligned}
& f_{1}\left(x_{1}, x_{2}, x_{3}, x_{4}, x_{5}\right)=\alpha\left(V_{s c}, n, \mu, r_{c}, h\right), \\
& f_{2}\left(x_{1}, x_{2}, x_{3}, x_{4}, x_{5}\right)=\Delta E\left(V_{s c}, n, \mu, r_{c}, h\right), \\
& f_{3}\left(x_{1}, x_{2}, x_{3}, x_{4}, x_{5}\right)=C\left(V_{s c}, n, \mu, r_{c}, h\right) .
\end{aligned}
$$

Thus, the final optimization problem can be summarized as a standard minimization:

$$
\operatorname{Min}\left\{\frac{1}{\alpha}, \Delta E, C\right\} \text {. }
$$

The problem is completed by adding constrains to the above optimization functions. In the proposed approach, two constrains were assumed:

(a) The limiting ratio $(\beta)$ is the ratio of the peak limited current to peak nominal current, $\beta \geq 2$, allowing the relays and fault detection systems to start.

(b) The normal ac losses $\left(P_{a c}\right)$ must not be greater than 15 watts. These constrains can be formulized as:

$$
g_{1}\left(x_{1}, x_{2}, x_{3}, x_{4}, x_{5}\right)=\beta\left(V_{s c}, n, \mu, r_{c}, h\right) \geq 2,
$$


Table 2. Limits of variables for optimization LSFCL.

\begin{tabular}{lcccc}
\hline \multicolumn{1}{c}{ Parameter } & Symbol & $\begin{array}{c}\text { Min } \\
\text { value }\end{array}$ & $\begin{array}{c}\text { Start } \\
\text { value }\end{array}$ & $\begin{array}{c}\text { Max } \\
\text { value }\end{array}$ \\
\hline HTS volume $\left(\mathrm{m}^{3}\right)$ & $V_{S C}$ & $10-3$ & $4 \times 10^{-3}$ & $10^{-2}$ \\
Turn ratio & $n$ & 80 & 120 & 150 \\
Permeability & $\mu$ & 50 & 65 & 80 \\
Core radius $(\mathrm{m})$ & $r_{c}$ & 0.38 & 0.4 & 0.48 \\
Core height $(\mathrm{m})$ & $h$ & 1 & 1.5 & 2 \\
\hline
\end{tabular}

Table 3. Parameters of optimization algorithm.

\begin{tabular}{lcc}
\hline \multicolumn{1}{c}{ Parameter } & Symbol & Value \\
\hline Maximum temperature for iteration & $T_{\max }$ & 5000 \\
Initial temperature for objectives & $T_{f i}$ & $10^{-4}-10^{4}$ \\
Decreasing factor & $r$ & 0.99 \\
Importance of constrains & $b$ & 5 \\
\hline
\end{tabular}

$$
g_{2}\left(x_{1}, x_{2}, x_{3}, x_{4}, x_{5}\right)=P_{a c}\left(V_{s c}, n, \mu, r_{c}, h\right) \leq 15 \text {. }
$$

Eventually, the problem can be defined as:

$$
\begin{aligned}
& \operatorname{Min}\left\{\frac{1}{\alpha}, \Delta E, C\right\} \\
& \Rightarrow \text { Subject to }:\left\{\begin{array}{l}
\frac{1}{\beta}-0.5 \leq 0 \\
P_{a c}-15 \leq 0
\end{array}\right.
\end{aligned}
$$

The limits of the selected variables and optimization parameters are shown in Tables 2 and 3, respectively. The mini and max values of variables were based on the stipulated and actual limiting performances [19].

In Table 3 , dimensionless parameters, i.e. $T_{\max }$, $T_{f i}, r$, and $b$, are maximum temperature of $\mathrm{SA}$, initial temperature of any objective, decreasing factor of temperature, and penalty factor (describing the importance of constrains), respectively.

To achieve optimal design of LSFCL, several iterations in each simulation have been performed and for any set of objective priorities, the most appropriate design is recognized. As the objective functions are not in the same dimensions, normalized probabilities for any functions and constraints can be achieved by FDLN or FNLD methods. For example, when all objectives have the same importance by equal annealing temperature, the variations of parameters, objectives, and constraints are presented in Figures 9 to 11 , respectively, for comparing FDLN and FNLD methods.

Considering nine priority levels and three objectives, Figure 12 shows 729 (i.e. $9^{3}$ ) round optimization process results for the objective functions resulted by changing the temperature of the inter-proportional objectives based on priority selection, i.e. $10^{4}$ (extremely

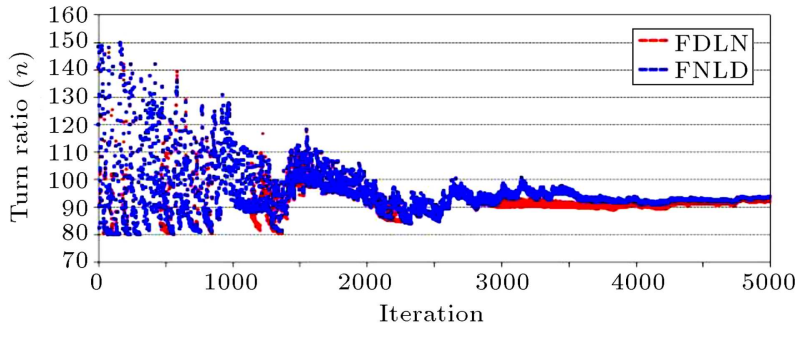

(a)

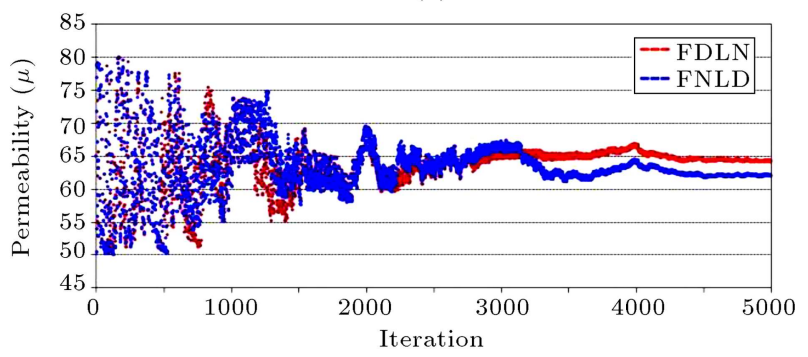

(b)

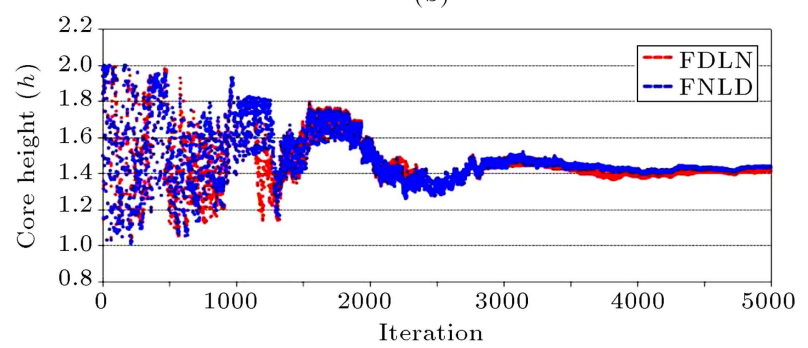

(c)

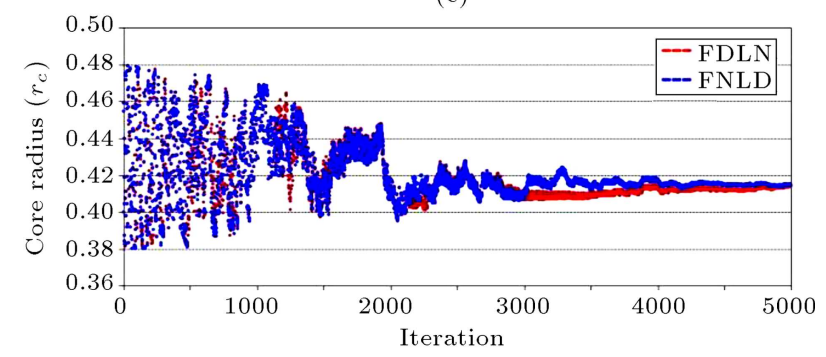

(d)

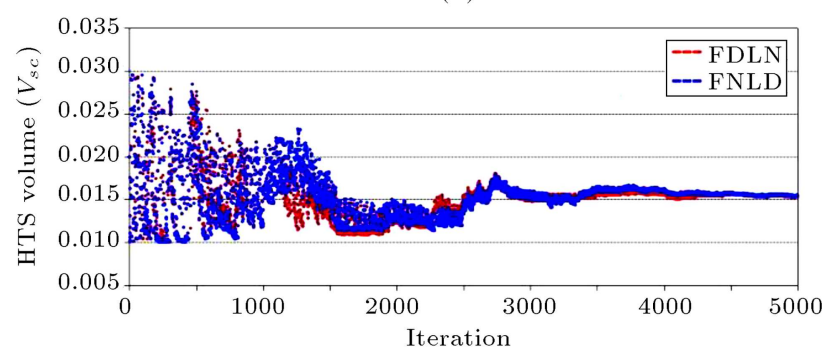

(e)

Figure 9. Comparison of design parameters in FDLN and FNLD for equal importance of objectives: (a) Turn ratio; (b) permeability; (c) core height; (d) core radius; and (e) HTS volume.

high), $10^{3}$ (very high), $10^{2}$ (high), $10^{1}$ (slightly high), $10^{0}$ (average), $10^{1}$ (slightly low), $10^{2}$ (low), $10^{-3}$ (very low), and $10^{-4}$ (extremely low), using FDLN technique. Figure 13 shows the related results for the FNLD method. In these figures, blue points are the final 


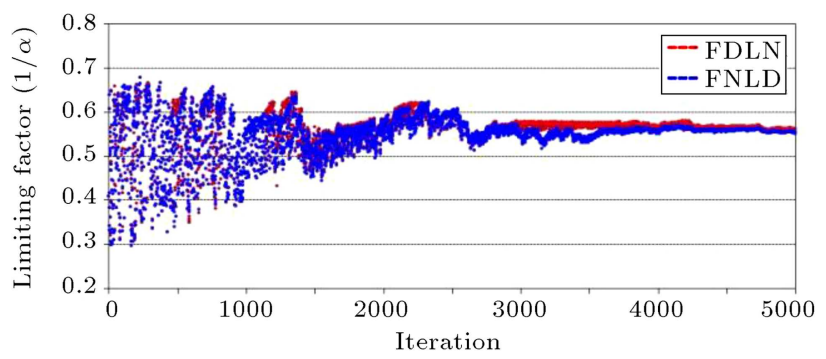

(a)

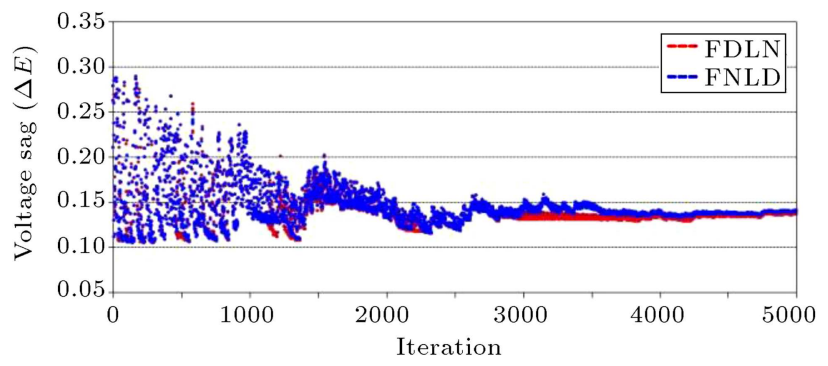

(b)

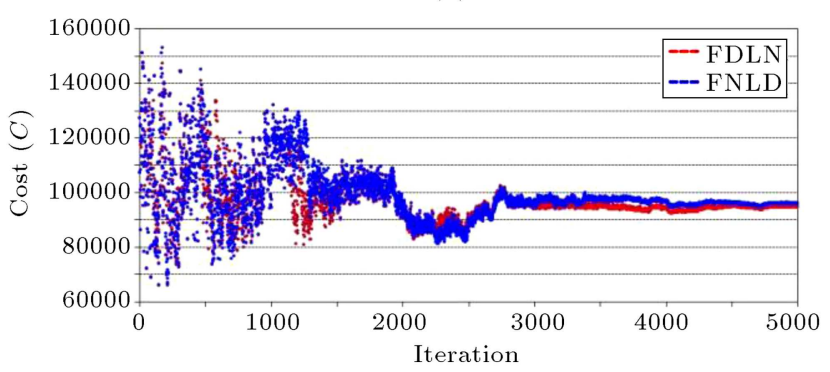

(c)

Figure 10. Comparison of objectives results in FDLN and FNLD for equal importance of objectives: (a) Limiting factor; (b) voltage sag; and (c) cost.

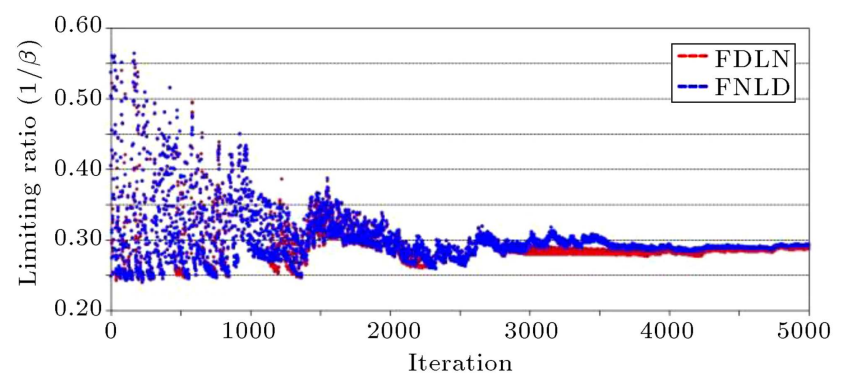

(a)

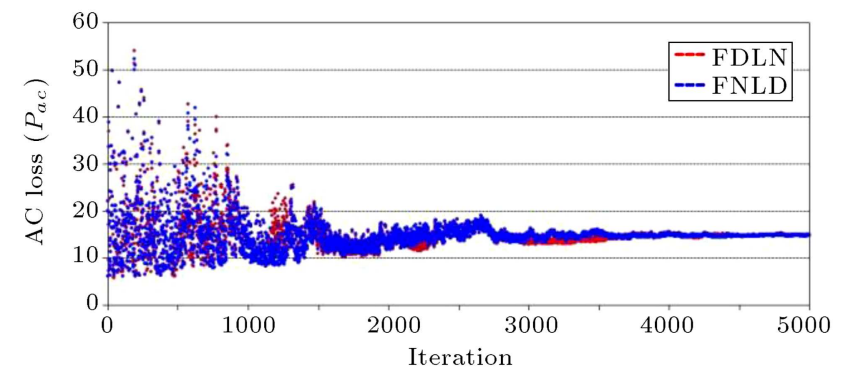

(b)

Figure 11. Comparison of constraints results in FDLN and FNLD for equal importance of objectives: (a) Limiting ratio; and (b) AC loss.

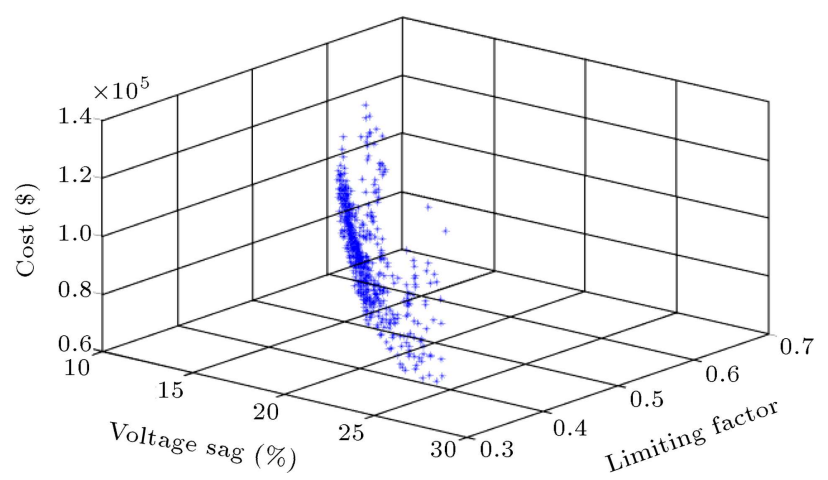

Figure 12. Final Pareto front based on FDLN method.

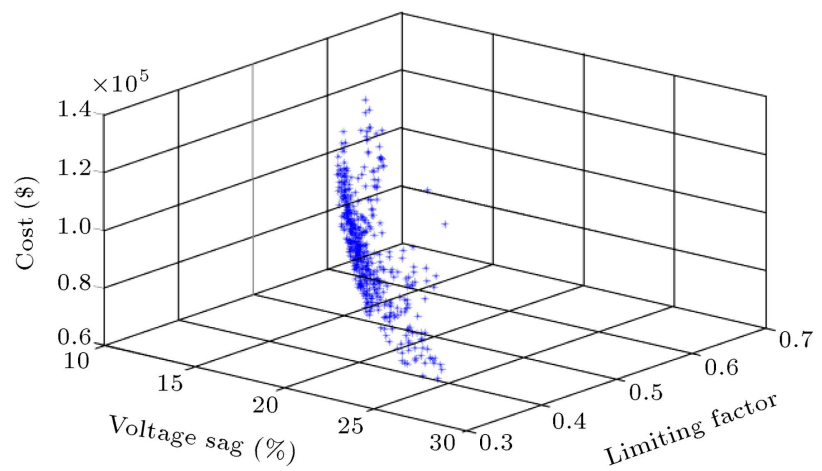

Figure 13. Final Pareto front based on FNLD method.

points which are not dominated by one another and each of them can be a solution for the purpose design of an LSFCL in a distribution system through the priority of the objective functions. As the Pareto fronts (Figures 12 and 13) present difference in only few points, these two methods are acceptably capable of finding Pareto points and drawing Pareto front. Further required points can be obtained by increasing ranking scale of the criteria higher than nine.

\section{Comparison of the two proposed methods}

Considering numerical results from simulations, as the first observation, the FNLD method is proportionally faster than another. While the FNLD algorithm is carried out in about 432 seconds by a Pentium IV $3.40 \mathrm{GHz}$, the FDLN takes 574 seconds, i.e. about $\% 30$ slower. In FNLD method, the penalty factor of constrains $(b)$ is more effective than that in FDLN. However, in a similar $b$, the FDNL produces enhanced cases. In both methods, increasing the importance of objectives $\left(T_{f i}\right)$ results in better answers.

\section{Conclusion}

In this paper, a novel heuristic approach for optimization of limiting devices based on multi-objective criteria decision making was described via two methods. This was accomplished by modeling an inductive super- 
conducting fault current limiter in PSCAD/EMTDC platform. A compromise between three optimal functions, i.e. current limiting factor, voltage sag, and economic construction cost, was extracted via nondominated solutions while considering two constraints, i.e. limiting ratio and ac loss. Both the proposed methods were compared and merits of each of them were discussed. However, the proposed methods were set for a particular case study; the designer can figure out exactly how much more cost is incurred on one objective depending on the priority of other objectives.

The heuristic optimization approach set out in this paper was for a particular case study. However, a designer must be capable of selecting any of the solutions lying on the Pareto front without jeopardizing optimality. More importantly, the designer can tell exactly how much more cost is incurred on a certain objective if another one is favored by a certain amount.

\section{References}

1. Henning, A. and Kurrat, M. "Thermal-electric simulations of coated conductors with a variable conductivity of the buffer layer", IEEE Trans. Appl. Supercond., $\mathbf{1 7}(2)$, pp. 3443-3446 (2007).

2. Noe, M. and Steurer, M. "High-temperature superconductor fault current limiters: concepts, applications, and development status", Superconductor Science and Technology, 20, pp. R15-R19 (2007).

3. Morandi, A., Bocchi, M., Fabbri, M., Martini, L., Negrini, F. and Ribani, P.L. "Comparison of the performances of three different types of fault current limiter in the distribution network", J. Phys.: Conf. Ser., 43, pp. 909-912 (2008).

4. Heydari, H., Faghihi, F., Sharifi, R. and Poursoltanmohammadi, A.H. "Superconducting technology for overcurrent limiting in a $25 \mathrm{kA}$ current injection system", Supercond. Sci. Technol., 21(9), p. 095016 (2008).

5. Yamaguchi, H., Kataoka, T. Yaguchi, K., Fujita, S., Yoshikawa, K. and Kaiho, K. "Characteristics analysis of transformer type superconducting fault current limiter", IEEE Trans. Appl. Supercond., 14(2), pp. 815818 (2004).

6. Kado, H., Ichikawa, M., Shibuya, M., Kojima, M., Kawahara, M. and Matsumura, T. "Inductive type fault current limiter using Bi-2223 thick film on $\mathrm{MgO}$ cylinder with Bi-2212 buffer Layer", IEEE Trans. Appl. Supercond., 15(2), pp. 2051-2054 (2005).

7. Kirkpatrick, S., Gelatt, C.D. and Vecchi, M.P. "Optimization by simulated annealing", Science, 220, pp. 671-680 (1983).

8. Geman, S. and Geman, D. "Stochastic relaxation, Gibbs distributions and the Bayesian restoration of images", IEEE Trans. Pattern Analysis and Machine Intelligence, 6, pp. 721-741 (1984).
9. Suman, B. "Simulated annealing-based multiobjective algorithms and their application for system reliability", Engineering Optimization, 35(4), pp. 391-416 (2003).

10. Dahm, T. and Scalapino, D.J. "Analysis and optimization of inter-modulation in high-Tc superconducting microwave filter design", IEEE Trans. Appl. Supercond., 8(4), pp. 149-157 (1998).

11. Noguchi, S., Yamashita, M., Yamashita, H. and Ishiyama, A. "An optimal design method for superconducting magnets using HTS tape", IEEE Trans. Appl. Supercond., 11(1), pp. 2308-2311 (2001).

12. Noguchi, S., Yamashita, M., Yamashita, H. and Ishiyama, A. "An optimization method for design of SMES coils using YBCO tape", IEEE Trans. Appl. Supercond., 13(2), pp. 1856-1859 (2003).

13. Han, S.I., Muta, I., Hoshino, T., Nakamura, T. and Maki, N. "Optimal design of superconducting generator using genetic algorithm and simulated annealing", IEE Proc. Elect. Power Appl., 151(5), pp. 543-544 (2004).

14. Sharifi, R. and Heydari, H. "Multiobjective optimization for HTS fault-current limiters based on normalized simulated annealing", IEEE Trans. Appl. Supercond., 19(4), pp. 3675-3682 (2009).

15. Heydari, H. and Sharifi, R. "Three-dimensional Pareto optimal design of inductive superconducting fault current limiters", IEEE Trans. Appl. Supercond., 20(5), pp. 2301-2311 (2010).

16. Osorio, R., Betrancourt, A., Francois, M.X., Veira, J.A. and Vidal, F.A. "A superconducting fault current limiter integrated in the cold heat exchanger of a thermo-acoustic refrigerator", Supercond. Sci. Technol., 21(9), p. 095013 (2008).

17. Kado, H. and Ichikawa, M. "Performance of a highTc superconducting fault current limiter-design of a $6.6 \mathrm{kV}$ magnetic shielding type superconducting fault current limiter", IEEE Trans. Appl. Supercond., 7(2), pp. 993-996 (1997).

18. Chen, M., Baumann, T., Unternahrer P. and Paul, W. "Fabrication and characterization of superconducting rings for fault current limiter application", Physica $C$, 282(7), pp. 2471-2472 (1997).

19. Paul, W., Baumann, T., Rhyner, J. and Platter, F. "Tests of $100 \mathrm{~kW}$ high-Tc superconducting fault current limiter", IEEE Trans. Appl. Supercond., 5(2), pp. 1059-1062 (1995).

20. Sokolovsky, V., Meerovich, V., Vajda, I. and Beilin, V. "Superconducting FCL: design and application", IEEE Trans. Appl. Supercond., 14(3), pp. 1990-2000 (2004).

21. Usoskin, A., Mumford, F., Dietrich, R., Handaze, A., Prause, B., Rutt, A. and Schlenga, K. "Inductive fault current limiters: Kinetics of quenching and recovery", IEEE Trans. Appl. Supercond., 19(3), pp. 1859-1862 (2009).

22. Meerovich, V. and Sokolovsky, V. "Thermal regimes of HTS cylinders operating in devices for fault current limitation", Supercond. Sci. Technol., 20, pp. 457-462 (2007). 
23. Ahn, M.C., Park, D.K., Yang, S.E., Kim, A.J., Kim, H.M., Kang, H., Nam, K., Seok, B.Y., Park, J.W. and Ko, T.K. "A study on the design of the stabilizer of coated conductor for applying to SFCL", IEEE Trans. on Appl. Supercond., 17(2), pp. 1855-1858 (2007).

24. Bean, P. "Magnetization of hard superconductors", Phys. Rev. Lett., 8, p. 250 (1962).

25. Norris, W.T. "Calculation of hysteresis losses in hard superconductors carrying ac: isolated conductors and edges of thin sheets", J. Phys. D. Appl. Phys., 3(4), pp.489-507 (1970).

26. Giese, R.F. and Runde, M. "Assessment study of superconducting fault-current limiters operating at 77 K", IEEE Trans. Power Delivery, 8(3), pp. 1138-1147 (1993).

27. Tappeta, R., Renaud, J. and Rodriguez, J. "An interactive multiobjective optimization design strategy for decision based multidisciplinary design", Engineering Optimization, 34(5), pp. 523-544 (2002).

28. Aggelogiannaki, E. and Sarimveis, H. "A simulated annealing algorithm for prioritized multiobjective optimization-implementation in an adaptive model predictive control configuration", IEEE Trans. Syst., Man, Cybern. B, Cybern, 37(4), pp. 902-915 (2007).

29. Suman, B. and Kumar, P. "A survey of simulated annealing as a tool for single and multiobjective optimization", Jour. Operational Research Society, 57, pp. 1143-1160 (2006).

30. Suman, B. "Study of simulated annealing based algorithms for multiobjective optimization of a constrained problem", Computers and Chemical Engineering, 28, pp. 1849-1871 (2004).

31. Bandyopadhyay, S., Saha, S., Maulik, U. and Deb, K. "A simulated annealing-based multiobjective optimization algorithm: AMOSA", IEEE Trans. Evol. Comp., 12(3), pp. 269-283 (2008).
32. Adiche, C. and Aider, M. "A hybrid method for solving the multi-objective assignment problem", Jour. Mathematical Modeling and Algorithms, 9(2), pp. 149164 (2010).

33. Ye, L., Majoros, M., Coombs, T. and Campbell, A. "System studies of the superconducting fault current limiter in electrical distribution grids", IEEE Trans. Appl. Supercond., 17(2), pp. 2339-2342 (2007).

\section{Biographies}

Reza Sharifi received the BSc degree in Electronics Engineering from University of Guilan, Rasht, Iran, in 1995, and the $\mathrm{MSc}$ and $\mathrm{PhD}$ degrees in Electrical Power from Iran University of Science and Technology (IUST) in 1998 and 2011, respectively. He is currently Assistant Professor at Islamic Azad University, West Tehran Branch. His research interests are the optimization method and applied superconductors in power systems and electrical safety management.

Hossein Heydari received the BSc degree in Electrical Engineering in 1985, the MSc degree in Power Electronics from Loughborough University in 1987, and the PhD degree in Transformer Core Losses from the University of Wales in 1993. He is currently Associated Professor at Iran University of Science and Technology (IUST) as an academic member of the Electrical Power Group. Also, he was appointed as the Director of the High Voltage and Magnetic Materials Research Center. His lecture duties are: electrical machines, electrical measurement and instrumentation, and EMC applications in electrical power systems. His research interests are in EMC considerations in electrical power systems, FCL, superconductors, and electrical machine core losses. 